\section{Differences in Psychopathology among Patients with Dual Diagnosis Receiving Treatment in Mental Health Services and Substance use Treatment Programs}

\section{Abstract}

Dual diagnosis is a commonly observed phenomenon in units addressing mental health and substance use problems. The purpose of this study was to examine the differences in psychopathology between patients with dual diagnosis receiving treatment for mental disorders in mental health services and those receiving treatment for substance use disorders in substance use treatment programs and to suggest possible predictive factors affecting symptoms of psychopathology. Participants were 80 individuals with dual diagnosis receiving treatment either for substance use disorders or for mental disorders in substance use treatment programs, emergency units, psychiatric hospitals and mental health rehabilitation units across Cyprus. The EuropASI and the SCL-90-R were administered individually to each one of the participants. Results revealed that patients with dual diagnosis receiving treatment in mental health services demonstrated higher symptoms of psychopathology regarding obsession-compulsion, interpersonal sensitivity, depression, anxiety, paranoid ideation and psychotism, in comparison to those receiving treatment in substance use treatment programs. Mental health service patients also exhibited higher severity of symptoms and higher distress because of the positive symptoms. Cannabis use in the last 30 days predicted higher levels of depression, anxiety, paranoid ideation and psychoticism, but lower GSI. Practical applications are analyzed.

Keywords: Addictions; Dual Diagnosis; Mental Health Services

\author{
Prodromou $\mathrm{M}$ and \\ Koukia E \\ Department of Nursing, National and \\ Kapodistrian University of Athens, \\ Greece
}

Corresponding author: Prodromou M

maria.prodromou@gmail.com

Department of Nursing, National and Kapodistria University of Athens, Greece.

Citation: Prodromou M, Koukia E. Differences in Psychopathology among Patients with Dual Diagnosis Receiving Treatment in Mental Health Services and Substance use Treatment Programs. Dual Diagn Open Acc. 2016, 1:1.

\section{Introduction}

Historically, the term "dual diagnosis" was first established in the USA during the 1980's. The use of this term in Europe started more recently. Dual diagnosis refers to cases of a) individuals suffering from an addictive disorder who also exhibit some psychiatric symptoms at the same time, b) individuals suffering from a serious mental disorder who also use psychoactive substances, c) individuals with co-occurring mental disorder and substance use disorder, and d) individuals that had both types of disorder at some point of their life, but not necessarily at the same time [1]. As Rostad and Checinski (1996) point out, the nature of this cooccurrence is complex, often causing confusion to the scientific community where no consensus is yet established regarding the concept of dual diagnosis [2].

The study of dual diagnosis as a phenomenon started during the late 1970's, when Woody and Blaine (1979) found a significant relationship between depression and substance use in individuals undergoing treatment for substance use [3-5]. During the decades that followed, numerous studies have shown that around $50-75 \%$ of individuals undergoing treatment for substance use suffered from a mental disorder at the same time. In addition, around 20$50 \%$ of individuals undergoing treatment for a mental disorder suffered from a substance use disorder as well [4]. More recently, Vergara-Moragues et al. (2012) examined the co-occurrence of mental disorders in cocaine dependent individuals, aged 20-55, who received treatment during the years 2008-2010 [6]. They found that more than $65 \%$ of the participants could be diagnosed with lifetime dual diagnosis, with $55.5 \%$ of them having a cooccurring Axis I disorder and $30.4 \%$ having a co-occurring Axis II disorder. Regarding Axis I disorders, mood disorders were the most common (31.3\%), followed by anxiety disorders $(23.8 \%)$, 
psychotic disorders (18.9\%) and eating disorders at a very low percentage (1.8\%). The most common Axis II disorder was antisocial personality disorder $(22.5 \%)$, followed by borderline personality disorder (14.1\%). Similarly, Arias et al. (2013) found that $74.4 \%$ of cocaine dependent outpatients could be diagnosed with dual diagnosis, with mood disorders and anxiety disorders as the most common co-occurring mental disorders [7]. High percentages of personality disorders were also reported, with antisocial personality disorder as the most common. Opioid dependents also exhibit high rates of co-occurring lifetime mood disorders, with major depressive disorder as the most frequent [8].

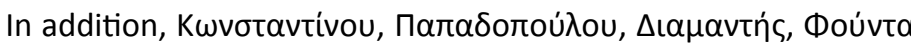

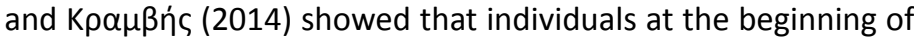
buprenorphine substitution therapy demonstrate high symptoms of psychopathology [9]. According to SCL-90-R scores, they exhibit high symptoms of somatization, obsession-compulsion, interpersonal sensitivity, depression, anxiety and paranoid ideation, along with high positive symptoms distress index. A large-scale study by Wu, Gersing, Swartz, Burchett, Li, and Blazer (2013) showed that $24.9 \%$ of individuals receiving treatment for mental health issues suffered from dual diagnosis [10]. The most common mental disorders in participants with dual diagnosis, were mood disorders (54.2\%), followed by anxiety disorders $(24.5 \%)$ and at lower percentages by personality disorders (13\%), psychotic disorders (11.1\%), adjustment disorder (7.3\%), dementia and other cognitive disorders (54\%), disorders usually first diagnosed in infancy, childhood or adolescence (4.8\%), somatoform disorders (2\%) and eating disorders (1.8\%). Moreover, Rady, Salama, Elkholy, and Shawky (2013a, 2013b, 2013c, 2013d) showed that $57.32 \%$ of personality disorders patients, $17.71 \%$ of mood disorders patients, $17.42 \%$ of anxiety disorders patients and $16.67 \%$ of schizophrenic patients of a psychiatric hospital of Egypt suffered from dual diagnosis. It is often observed that patients with dual diagnosis present more than one mental disorder along with the substance use disorder (e.g., Ding, Yang, Cheng, Schiltz, summers, and Skinstad, 2011; Lechner et al., 2013) [11-16].

In an interesting study, Havassy, Alvidrez, and Owen (2004) compared dual diagnosis patients receiving treatment either in mental health services or in substance use treatment programs [17]. They reported that $60 \%$ of all patients in mental health services suffered from dual diagnosis, while $49 \%$ of substance use treatment program patients had dual diagnosis. It is important to note that this difference between the two percentages was statistically significant. Regarding the rates of specific disorders, schizophrenia and other psychotic disorders, as well as mood disorders were higher among mental health service patients, while anxiety disorders were higher among substance use treatment program patients.

Regarding the causal links between the two disorders in dual diagnosis, there are four general hypotheses stated in the relevant literature (Kushner and Mueser, 1993; Mueser et al., 1998):

a) Addiction to psychoactive substances can cause one or more symptoms of mental disorders.

b) Mental disorders may lead to addiction to psychoactive substances. c) The two disorders are caused by a common etiological factor, such as brain dysfunction, genetic predisposition, early exposure to stressful events or traumas.

d) The mental disorder and substance use interact and maintain each other.

Studies show that patients with dual diagnosis demonstrate poorer prognosis than patients with a single diagnosis $[18,19]$. Moreover, it appears that the length of stay at therapy is significantly less in the case of individuals with dual diagnosis $[15,19]$, while the use of health services and the frequency of hospitalizations are significantly higher [20,21]. In addition individuals with dual diagnosis demonstrate higher suicide rates $[20,22]$, as well as higher conviction rates [23] and imprisonment rates (Drake and Muesser, 2000). Ding et al. (2011) suggested that the mental health status of an individual could be an important predictive factor for the length of stay in therapy. Thus, considering the serious consequences of dual diagnosis and the significance of effective treatment methods, an initial assessment of mental health status is vitally important in units providing treatment for patients with dual diagnosis [15]. It is important to note here that individuals with dual diagnosis often receive treatment either in mental health services or substance use treatment programs, where it is possible that they receive treatment for only one of the two diagnoses, or for both. The last few years, professionals in mental health services as well as substance use treatment programs of Cyprus have noticed an increase of individuals with dual diagnosis receiving treatments in mental health services and substance use treatment programs. This phenomenon raises concerns to the professionals regarding differential diagnosis and efficient treatment methods aiming at harm reduction. Therefore, research on the differences in mental health characteristics regarding patients in mental health services and patients in substance use treatment programs is much needed.

The purpose of this study is a) to examine the differences in psychopathology between patients with dual diagnosis receiving treatment for mental disorders in mental health services and those receiving treatment for substance use disorders in substance use treatment programs, and b) to suggest possible predictive factors affecting psychopathology. Moreover, this study aims to promote the development of a dual diagnosis knowledge database in Cyprus.

\section{Materials and Methods}

\section{Participants and data collection}

Participants were 80 individuals with dual diagnosis $(77.5 \%$ males and $22.5 \%$ females), aged $18-70$, receiving treatment either for substance use disorders or for mental disorders in substance use treatment programs, emergency units, psychiatric hospitals and mental health rehabilitation units across Cyprus. They were grouped into two categories: mental health service patients $(n=40)$ and substance use treatment program patients $(n=40)$. Dual diagnosis treatment was not provided to any of the participants.

The questionnaires were administered individually by the 
researcher, at the treatment units. Data were collected from January to March 2014, as part of a larger study for the needs of the researcher's doctoral thesis. The researcher visited the treatment units twice a week during the data collection period and interviewed all patients with dual diagnosis who were present at the units. The study was explained in detail and informed consent was requested in order to continue to the completion of the questionnaires.

\section{Inclusion and exclusion criteria}

Inclusion and exclusion criteria for participants were as follows:

- Participant must be sober during the assessment.

- Participant must be able to provide data which meet the quality standards necessary for data analysis

Processes.

- $\quad$ Participant must not be in a psychological state that puts them or other people in danger.

- $\quad$ Participant must not suffer from any brain damage or any cognitive or mental disorder due to a general

medical condition.

- Participant must be over 18 and under 70 years old.

\section{Instruments}

European addiction Severity Index- EuropASI [24]. EuropASI is an adaption of the Addiction severity index - ASI $5^{\text {th }}$ Edition for the European population. The purpose of this instrument is to determine the severity of addiction to psychoactive substances [25]. It is a semi-structured interview, containing 30 questions, mainly used for diagnostic purposes and assessment of treatment outcome. The instrument consists of eight domains: general information, medical status, chemical use, employment/ support, family/social relationships, legal status, and psychiatric/ psychological status [25]. EuropASI is considered as a valid and reliable tool for assessing the severity of substance use in psychiatric populations. Test-retest reliability has been reported at 0.89 . It has been translated in Greek and used in Cyprus as well by trained interviewers [26]. In this study, the first domain of EuropASI (General Information) is used for the collection of information regarding the medical and legal history of participants.

\section{Symptom Checklist- 90-Revised (CL- 90-R)}

Designed by Derogatis (1994), this instrument assesses psychological problems and identifies symptoms of psychopathology [27]. It consists of 90 items rated on a 5-point scale, assessing psychiatric disturbance in nine dimensions: somatization, obsession-compulsion, interpersonal sensitivity, depression, anxiety, hostility, phobic anxiety, paranoid ideation and psychotism. In addition, three global indices of distress are calculated based on the nine dimensions: the General Severity
Index (GSI), which reflects the extend of the current psychiatric disturbance, the Positive Symptom Total (PST), which reflects the number of questions rated above 1 point, and the Positive Symptom Distress Index (PSDI), which reflects the intensity of the symptoms. SCL-90-R has been successfully used to measure the psychological functioning of substance use populations. It is considered as a highly valid instrument $[28,29]$. Studies reported high levels of internal consistency for each of the nine dimensions, as well as high test-retest reliability [28]. This study uses the Greek version of SCL-90-R, which is also considered as a valid and reliable measure.

\section{Data collection}

The Statistical Package for Social Sciences (SPSS) version 20.0 was used for data analysis. Descriptive statistics were used for demographic characteristics and t-test was used to determine the differences in psychopathology between patients in mental health services and patients in substance use treatment programs. Furthermore, logistic regression analysis was conducted in order to determine factors affecting psychopathology.

\section{Results}

\section{General information}

Table 1 presents some general medical and legal information of the participants of each type of unit. As can be seen, mental health service ( $\mathrm{mhu}$ ) patients present higher means in comparison to substance use treatment program (du) patients regarding number of diagnoses (mhuM=3.03, duM=2.55), number of hospitalizations ( $m$ huM $=12.25$, duM=7.73), number of outpatient visits for psychological problems ( $\mathrm{mhuM}=63.02$, $\mathrm{duM}=18.78$ ) and prison time ( $\mathrm{mhuM}=5.55$, duM=2.10). Mean age of first substance use for males is 17.88 in mental health services and 17.52 in substance use treatment programs. For females, mean age is 17.50 in mental health services and 17.60 in substance use treatment programs (Table 2). The main substance used in most cases is cannabis, followed by cocaine (Table 3), both in mental health services (57.5\% cannabis, $20 \%$ cocaine) and substance use treatment programs $(30 \%$ cannabis, $20 \%$ cocaine).

Table 1: Means and standard deviations regarding the number of diagnoses, number of hospitalizations, number of outpatient visits for psychological problems and prison time of participants according to the type of unit.

\begin{tabular}{lcc|} 
& \multicolumn{2}{c}{ Type of unit } \\
& $\begin{array}{c}\text { Mental health services } \\
\text { Mean (SD) }\end{array}$ & $\begin{array}{c}\text { Substance use } \\
\text { treatment } \\
\text { programs } \\
\text { Mean (SD) }\end{array}$ \\
\hline $\begin{array}{l}\text { Number of diagnoses (1-5) } \\
\text { Number of hospitalizations }\end{array}$ & $3.03(0.72)$ & $2.55(0.78)$ \\
\hline $\begin{array}{l}\text { Number of outpatient visits } \\
\text { for psychological problems }\end{array}$ & $12.25(25.28)$ & $7.73(12.71)$ \\
\hline Prison time (in months) & $63.02(76.47)$ & $18.78(50.68)$ \\
\hline & $5.55(13.30)$ & $2.10(8.70)$ \\
\hline
\end{tabular}


Table 2: Means and standard deviations regarding age of first substance use according to type of unit.

\begin{tabular}{l|cc|} 
Gender & \multicolumn{2}{c}{ Type of unit } \\
Mental health services & $\begin{array}{c}\text { Substance use treatment } \\
\text { programs Mean (SD) }\end{array}$ \\
\hline Male & $17.88(2.67)$ & $17.52(3.51)$ \\
\hline Female & $17.50(8.69)$ & $17.60(4.28)$ \\
\hline Total & $17.76(5.22)$ & $17.53(3.56)$
\end{tabular}

Table 3: Cross tabulations of main substances used according to type of unit.

\begin{tabular}{|c|c|c|c|c|}
\hline & \multicolumn{2}{|c|}{ Type of unit } & \multirow[b]{2}{*}{ Total } \\
\hline & & $\begin{array}{l}\text { Mental } \\
\text { health } \\
\text { services }\end{array}$ & $\begin{array}{c}\text { Substance use } \\
\text { treatment } \\
\text { programs }\end{array}$ & \\
\hline \multirow{10}{*}{$\begin{array}{l}\text { Main } \\
\text { substance }\end{array}$} & Alcohol & $7.5 \%$ & $10.0 \%$ & $8.8 \%$ \\
\hline & Heroine & $2.5 \%$ & $15.0 \%$ & $8.8 \%$ \\
\hline & Methadone & $2.5 \%$ & $5.0 \%$ & $3.8 \%$ \\
\hline & Hallucinogens & $5.0 \%$ & $0.0 \%$ & $2.5 \%$ \\
\hline & Cocaine & $20.0 \%$ & $20.0 \%$ & $20.0 \%$ \\
\hline & Amphetamines & $2.5 \%$ & $7.5 \%$ & $5.0 \%$ \\
\hline & Cannabis & $57.5 \%$ & $30.0 \%$ & $43.8 \%$ \\
\hline & Alcohol and Drugs & $0.0 \%$ & $2.5 \%$ & $1.3 \%$ \\
\hline & Multiple Substances & $0.0 \%$ & $7.5 \%$ & $3.8 \%$ \\
\hline & Inhalants & $2.5 \%$ & $0.0 \%$ & $1.3 \%$ \\
\hline
\end{tabular}

T-test results between SCL-90-R and type of unit

The comparison of scores in all dimensions and the three global indices on SCL-90-R between participants from mental health services and those from substance use treatment programs showed that patients with dual diagnosis of mental health services demonstrated significantly higher scores in the dimensions of obsession-compulsion, interpersonal sensitivity, depression, anxiety, paranoid ideation and psychotism. Moreover, GSI and PSDI were also significantly higher in the mental health services group (Table 4).

\section{Logistic regression analysis results}

The association between Type of Unit and the 10 dimensions of the SCL-90-R was investigated using logistic regression analysis (Table 5).The results revealed that patients of the substance use treatment programs presented lower levels of paranoid ideation and psychoticism. An additional logistic regression analysis was conducted to examine the association between Use of cannabis in the last 30 days and the 10 dimensions of the SCL-90-R (Table-6). The results revealed that patients that used cannabis in the last 30 days had higher levels of depression, anxiety, paranoid ideation and psychoticism, but lower GSI.

\section{Discussion}

This study aimed to examine the differences in psychopathology between patients with dual diagnosis receiving treatment for mental disorders in mental health services and those receiving treatment for substance use disorders in substance use treatment programs and to suggest possible predictive factors affecting psychopathology. It was found that mental health service patients had significantly higher obsession-compulsion, interpersonal sensitivity, depression, anxiety, and paranoid ideation and psychotism symptoms in comparison to substance use treatment program patients. Moreover, they presented higher severity of symptoms and higher distress because of the positive symptoms. The results of the logistic regression analysis also confirmed that patients of the substance use treatment programs presented lower levels of paranoid ideation and psychoticism. This finding is partly in line with Havassy et al. (2004), who revealed higher rates of schizophrenia and other psychotic disorders, as well as mood disorders in mental health service patients. However, in contrast with the present study, they found that anxiety disorders were higher among substance use treatment program patients [17]. Cannabis use in the last 30 days predicted higher levels of depression, anxiety, paranoid ideation and psychoticism, but lower GSI in the present study. This result should raise concerns in the scientific community since the main substance used in most cases was cannabis, followed by cocaine, both in mental health services and substance use treatment programs. Researchers suggest that cannabis use may facilitate the onset of schizophrenia and other psychotic disorders in predisposed individuals at a younger age [30,31]. According to $\mathrm{Wu}$ et al. (2013), cannabis is the main substance used by patients with dual diagnosis in mental health services at the age range of 1824 , while cocaine is the main substance used at the age range of 25-44 [10].

Mean age of first substance use was around 17 and did not differ

Table 4. SCL-90-R scores of participants according to type of unit.

\begin{tabular}{|c|c|c|c|c|}
\hline \multicolumn{5}{|c|}{ Type of unit } \\
\hline Dimension & $\begin{array}{l}\text { Mental } \\
\text { health } \\
\text { services } \\
\text { Mean (SD) }\end{array}$ & $\begin{array}{l}\text { Substance } \\
\text { use } \\
\text { treatment } \\
\text { programs } \\
\text { Mean (SD) }\end{array}$ & $\mathbf{T}$ & $p$ value \\
\hline Obsession-compulsion & $2.07(0.85)$ & 1.46 (1.09) & 2.80 & 0.006 \\
\hline Interpersonal sensitivity & $2.03(0.77)$ & $1.28(0.98)$ & 3.77 & 0.000 \\
\hline Depression & $2.48(0.74)$ & $1.67(1.22)$ & 3.57 & 0.001 \\
\hline Anxiety & $1.72(0.98)$ & $1.21(1.06)$ & 2.23 & 0.028 \\
\hline Paranoid ideation & $2.91(0.84)$ & $1.84(1.10)$ & 4.92 & 0.000 \\
\hline Psychoticism & $2.25(0.94)$ & $0.98(0.91)$ & 6.11 & 0.000 \\
\hline GSI & $1.94(0.63)$ & $1.29(0.90)$ & 3.74 & 0.000 \\
\hline PSDI & $3.58(0.39)$ & $2.64(0.88)$ & 6.10 & 0.000 \\
\hline
\end{tabular}

Table 5. Logistic analysis regression results: Association between Type of Unit and the 10 dimensions of the SCL-90-R.

\begin{tabular}{|l|c|c|c|c|c|c|}
\hline & B & S.E. & p value & Exp(B) & \multicolumn{2}{c}{$95 \%$ C.I.for EXP(B) } \\
\hline & & & & & Lower & Upper \\
\hline Paranoid Ideation & -0.725 & 0.374 & 0.052 & 0.485 & 0.233 & 1.008 \\
\hline Psychoticism & -1.561 & 0.460 & 0.001 & 0.210 & 0.085 & 0.517 \\
\hline
\end{tabular}

Table 6. Logistic analysis regression results: Association between Use of cannabis in the last 30 days and the 10 dimensions of the SCL-90-R

\begin{tabular}{|c|c|c|c|c|c|c|}
\hline & \multirow{2}{*}{ B } & \multirow{2}{*}{ S.E. } & \multirow{2}{*}{$p$ value } & \multirow{2}{*}{$\operatorname{Exp}(B)$} & \multicolumn{2}{|c|}{ 95\% C.I.for EXP(B) } \\
\hline & & & & & Lower & Upper \\
\hline Depression & 2.428 & 0.810 & 0.003 & 11.337 & 2.319 & 55.429 \\
\hline Anxiety & 1.851 & 0.814 & 0.023 & 6.364 & 1.291 & 31.365 \\
\hline Paranoid Ideation & 1.016 & 0.405 & 0.012 & 2.761 & 1.249 & 6.104 \\
\hline Psychoticism & 1.225 & 0.482 & 0.011 & 3.405 & 1.325 & 8.753 \\
\hline GSI & -6.788 & 2.039 & 0.001 & 0.001 & 0.000 & 0.061 \\
\hline
\end{tabular}


significantly across units or gender. However, the majority of participants in this study are males, revealing that dual diagnosis is more frequent in males, which is also evident in other studies [32]. Furthermore, it appears that mental health patients often present more than one mental health disorder. Similar results concerning patients with dual diagnosis are evident in other studies as well, without reporting any comparison of mental health service and substance use treatment program patients. For example, Lechner et al. (2013) showed that more than a third of patients with dual diagnosis report more than one mental health disorder [16]. Ding et al. (2011) also revealed high rates of more than one disorder [15].

Participants of this study demonstrated high rates of hospitalizations, outpatient visits for psychological problems and prison time, with mental health service patients presenting higher means in these characteristics. Outpatient visits were almost more than three times higher and prison time was about twice as high in the case of mental health patients. There are numerous studies showing that the use of health services, frequency of hospitalizations [20,21], and conviction rates and imprisonment rates are generally higher in patients with dual diagnosis [22,23]. However, the comparison of mental health patients and substance use treatment program patients was not given any emphasis in the relevant literature in previous years.

The results of this study emphasize the need for further investigation regarding the characteristics and the differences of psychopathology between patients with dual diagnosis receiving treatment in mental health services and those receiving treatment in substance use treatment programs. Special emphasis should be given to the study of the association between cannabis use and psychopathology. The investigation of interaction mechanisms between the mental disorder and the substance use disorder in individuals with dual diagnosis, as well as their effects on the course of treatment, is vitally important.

\section{Conclusion}

This is the first study to compare specific symptoms of psychopathology among patients with dual diagnosis of mental health services and substance use treatment programs. Overall, this study showed that patients with dual diagnosis receiving treatment in mental health services demonstrated higher symptoms of psychopathology regarding obsession-compulsion, interpersonal sensitivity, depression, anxiety, paranoid ideation and psychotism, in comparison to those receiving treatment in substance use treatment programs. Mental health service patients also exhibited higher severity of symptoms and higher distress because of the positive symptoms. Cannabis use in the last 30 days appeared to predict higher levels of depression, anxiety, paranoid ideation and psychoticism in both types of unit. On a practical level, the results of this study emphasize the need of an initial assessment policy regarding psychopathology in individuals receiving detoxification and symptoms of substance use in individuals receiving treatment for mental disorders. Individuals with dual diagnosis are in need for a specialized treatment approach, requiring the differentiation of programs offered by mental health services and substance use treatment programs. The relevant literature suggests the development of integrated therapies, which have demonstrated significant results in decreasing rates of hospitalizations, service utilization, legal problems and convictions suggest the utilization of a combination of treatments, such as psychotherapy, cognitive and pharmacological interventions [33,34]. Dual diagnosis therapy programs should be tailored according to the individual needs of each patient and should be provided on a continuous basis in both mental health and substance use treatment programs. The effective application of such programs requires continuous and efficient communication between the two types of units, as well as specialized training of health professionals. In addition, this study highlights the need for further research on the subject, as well as the necessity of the development of a dual diagnosis knowledge database in Cyprus.

\section{Limitations}

This study did not address causal relationships between psychopathology and substance use. Factors contributing to the differences revealed were not examined. Differences in psychopathology may precipitate the onset of substance use or the onset of a fully diagnosable mental health disorder, or substance use may lead to symptoms of psychopathology and to the development of a mental health disorder. Another limitation is that results are solely based on a self-report instrument, not combined with other research methods. However, the questionnaire used in this study is considered as a valid and highly reliable instrument. 


\section{References}

1 Rassool GH (ed.) (2006) Understanding dual diagnosis: An overview. In: Dual Diagnosis Nursing, pp. 3-15.

2 Rostad P, Checinski K (1996) Dual diagnosis: Facing the challenge. Surrey: Wynne Howard Books.

3 Woody GE, Blaine J (1979) Depression in narcotic addicts: Quite possibly more than a chance association. In: Dupont R, Goldstein A O'Donnell J (Eds.), Handbook of Drug Abuse Rockville, MD: National Institute on Drug Abuse, pp. 277-285.

4 Sacks S, Ries RK (2005) Substance abuse treatment for persons with co-occurring disorders. USA: Center for Substance Abuse Treatment.

5 Flynn PM, Brown BS 2008 Co-occurring disorders in substance abuse treatment: Issues and prospects. Journal of Substance Abuse Treatment 34: 36-47.

6 Vergara-Moragues E, González-Saiz F, Lozano MO, Betanzos Espinosa P, Fernández Calderón F, et al. (2012) Psychiatric comorbidity in cocaine users treated in therapeutic community: Substance-induced versus independent disorders. Psychiatry Research 200: 734-741.

7 Arias F, Szerman N, Vega P, Mesias B, Basurte I, et al. (2013) Cocaine abuse or dependency and other psychiatric disorders. Madrid study on dual pathology. Revista de Psiquiatría y Salud Mental (English Edition) 6: 121-128.

8 Savant JD, Barry DT, Cutter CJ, Joy MT, Dinh A, et al. (2013) Prevalence of mood and substance use disorders among patients seeking primary care office-based buprenorphine/naloxone treatment. Drug and Alcohol Dependence 127: 243-247.

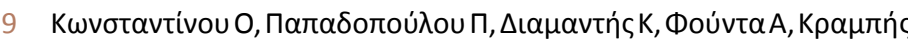

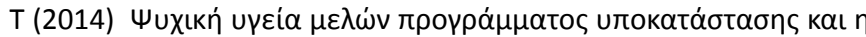

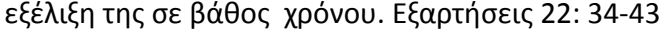

10 Wu LT, Gersing KR, Swartz MS, Burchett B, Li T K, et al. (2013) Using electronic health records data to assess comorbidities of substance use and psychiatric diagnoses and treatment settings among adults. Journal of Psychiatric Research 47: 555-563.

11 Rady A, Salama H, Elkholy O, Shawky A (2013) (a) Dual diagnosis of schizophrenia and substance abuse: the need for awareness European Psychiatry28: 791

12 Rady A, Salama H, Elkholy O, Shawky A (2013) (b) Dual diagnosis of mood disorders and substance abuse: the need for awareness. European Psychiatry 28: 792.

13 Rady A, Salama H, Elkholy O, Shawky A (2013) (c) Dual diagnosis of personality disorders and substance abuse: the need for awareness. European Psychiatry 28: 793.

14 Rady A, Salama H, Elkholy O, Shawky A (2013) (d) Dual diagnosis of personality disorders and substance abuse: the need for awareness. European Psychiatry 28: 845.

15 Ding K, Yang J, Cheng G, Schiltz T, Summers KM, et al. (2011) Hospitalizations and hospital charges for co-occurring substance use and mental disorders. Journal of Substance Abuse Treatment 40: 366-375.

16 Lechner WV, Dahne J, Chen KW, Pickover A, Richards JM (2013) The prevalence of substance use disorders and psychiatric disorders as a function of psychotic symptoms. Drug and Alcohol Dependence 131: 78-84.

17 Havassy BE, Alvidrez J, Owen KK (2004) Comparisons of patients with comorbid psychiatric and substance use disorders: implications for treatment and service delivery. American Journal of Psychiatry 161: 139-145.

18 Mericle AA, Ta Park VM, Holck P, Arria AM (2012) Prevalence, patterns, and correlates of co-occurring substance use and mental disorders in the United States: variations by race/ethnicity. Comprehensive Psychiatry 53: 657-665.

19 Schmidt LM, Hesse M, Lykke J (2011) The impact of substance use disorders on the course of schizophrenia-A 15-year follow-up study: Dual diagnosis over 15 years. Schizophrenia Research 130: 228-233.

20 Kessler RC, McGonagle KA, Zhao S, Nelson CB, Hughes M, et al. (1994) Lifetime and 12-month prevalence of DSM-III-R psychiatric disorders in the United States: results from the National Comorbidity Survey. Archives of General Psychiatry 51: 8-9.

21 Wu LT, Kouzis AC, Leaf PJ (1999) Influence of comorbid alcohol and psychiatric disorders on utilization of mental health services in the National Comorbidity Survey. American Journal of Psychiatry 156: 1230-1236.

22 Drake RE, Mueser KT (2000) Psychosocial approaches to dual diagnosis. Schizophrenia Bulletin 26: 105-118.

23 Piselli M, Elisei S, Murgia N, Quartesan R, Abram KM (2009) Cooccurring psychiatric and substance use disorders among male detainees in Italy. International Journal of Law and Psychiatry 32: 101-107.

24 Blacken P, Hendriks V, Pozzi G, Tempesta E, Hartgers C, et al. (1994) European addiction severity index (EuropASI) 5th ed. Athens: EMCCDA.

25 McLellan AT, Kushner H, Metzger D, Peters R, Smith I, et al. (1992) The fifth edition of the Addiction Severity Index. Journal of Substance Abuse Treatment 9: 199-213.

26 Kokkevi A, Hartgers C (1995) EuropASI: European adaptation of a multidimensional assessment instrument for drug and alcohol dependence. European Addiction Research 1: 208-210.

27 Derogatis L. R (1994) SCL-90-R administration, scoring and procedures manual. Minneapolis: NSC Pearson.

28 Horowitz LM, Rosenberg SE, Baer BA, Ureño G, Villaseñor VS (1988) Inventory of interpersonal problems: psychometric properties and clinical applications. Journal of Consulting and Clinical Psychology 56: 885-892.

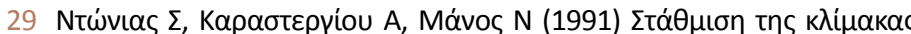

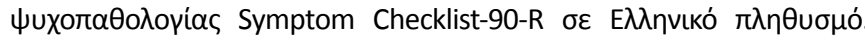

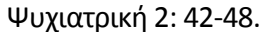

30 Degenhardt L, Hall W (2002) Cannabis and psychosis. Current Psychiatry Reports 4: 191-196.

31 Van Dijk D, Koeter MW, Hijman R, Kahn RS, van Den Brink W (2012) Effect of cannabis use on the course of schizophrenia in male patients: a prospective cohort study. Schizophrenia Research 137: 50-57.

32 Najt P, Fusar-Poli P, Brambilla P (2011) Co-occurring mental and substance abuse disorders: A review on the potential predictors and clinical outcomes. Psychiatry Research 186: 159-164.

33 Mangrum LF, Spence RT, Lopez M (2006) Integrated versus parallel treatment of co-occurring psychiatric and substance use disorders. Journal of Substance Abuse Treatment 30: 79-84.

34 Kelly TM, Daley DC, Douaihy AB (2012) Treatment of substance abusing patients with comorbid psychiatric disorders. Addictive Behaviors 37: 11-24. 